\title{
Wearable Coaching System
}

\section{Nur Alia Athirah Hj Mohtadzar ${ }^{1 *}$, Dyg Norkhairunnisa Abang Zaidel ${ }^{1}$, Abdul Rahman Kram', Kasumawati Lias ${ }^{1}$, Shahrol Mohamaddan', Mohamad Shaiful Abdul Karim $^{2}$, Yon Syafni Samat ${ }^{3}$, Shigeru Takayama ${ }^{4}$}

\author{
${ }^{1}$ Faculty of Engineering \\ Universiti Malaysia Sarawak, Kota Samarahan, Sarawak, 94300, MALAYSIA \\ ${ }^{2}$ Faculty of Electrical and Electronics Engineering \\ Universiti Malaysia Pahang, Pekan, Pahang, 26600, MALAYSIA \\ ${ }^{3}$ Faculty of Built Environment \\ Universiti Malaysia Sarawak, Kota Samarahan, Sarawak, 94300, MALAYSIA
}

${ }^{4}$ Faculty of Science and Engineering

Ritsumeikan University, Kusatsu, Shiga 525-8577, JAPAN

*Corresponding Author

DOI: https://doi.org/10.30880/ijie.2020.12.018

Received 8 April 2020; Accepted 2 June 2020; Available online 2 July 2020

\begin{abstract}
Wearable Coaching System is a multi-sensor device for real-time training coaching. The system comprises of six modules which are shoulder, waist, a pair of wrist and a pair of ankle modules. It is compact, light, comfortable to wear and is suitable for any size of user. It system has five main embedded sensors: heart rate, three-axis acceleration, gyro, temperature and blood oxygen saturation ( $\mathrm{SpO} 2)$ sensor. It operates in streaming mode for realtime data processing using two telecommunication tools: ZigBee and Bluetooth and low energy interface for recording mode is using an internal flash memory. In this paper, a wearable coaching system is designed to help athlete or trainer to perform proper and efficient exercise suitable to one's body needs. It measures the parameter of the body, analyze and provide feedback in a form of an advice. In order to improve the system, arm gesture mode performed by the wrist modules of the system is applied. This mode is to enable user to manage their exercising level to a more appropriate pace, suitable with their individual body by reproducing the feedback. In a supervised coaching training, percentage of heart rate threshold target is increasing, hence, producing a greater performance of training. It is proven in this paper, that wearable coaching system performs an accurate measurement in enhancing sports and coaching training, adding value to novel lifestyle exercising and health monitoring.
\end{abstract}

Keywords: Coaching, monitoring, exercise, heart rate, wearable, sensor, ZigBee, bluetooth

\section{Introduction}

A wearable coaching system is a combination of wireless sensor interacting with each other and actuators in order to coach an athlete training performances. [1] Lack or no monitoring of moderate activity or exercises such as walking, running or jogging could potentially pose serious threats to the human body. For instance, the incorrect body postures or excessive running could strain the body and lead to health problems such as back pain, slipped disks or knee pain. [4] One of the ways to minimize the health risks, is by designing a wearable system to monitor the body parameters 